\title{
A Hydromagnetic Boundary Layer Micropolar Fluid over a Stretching Surface in a Non- Darcian Medium with Permeability
}

\author{
Yomi Monday Aiyesimi, Abdulhakeem Yusuf*, Jiya Mohammed
}

Department of Mathematics and Statistics, Federal University of Technology, PMB 65, Minna, 00176-0000 Nigeria, Niger State, Nigeria *Corresponding Author: yusuf.abdulhakeem@futminna.edu.ng

Copyright (C) 2013 Horizon Research Publishing All rights reserved.

\begin{abstract}
Hydromagnetic boundary layer micropolar fluid flow over a stretching surface embedded in a non-Dacian medium with variable permeability was considered in this work. The governing partial differential equations were transformed into their equivalent cylindrical coordinate system from its original form (rectangular form). A set of similarity parameters are employed to convert the governing partial differential equations to ordinary differential equations. The obtained self-similar equations are solved using the Adomian Decomposition Method. The effect of various physical parameters on the velocity profile, microrotation and temperature distribution were investigated. The obtained results shows that as the magnetic parameter $R$ and the inverse Darcy number $D^{-1}$ increase the velocity profile and the microrotation reduce while the temperature profile increases.
\end{abstract}

Keywords Adomian method, Hydromagnetic, Micropolar, Permeability, Non Darcian

\section{Introduction}

The production of sheeting material, which includes both metal and polymer sheets, arises in a number of industrial manufacturing processes. The fluid dynamics due to a continuous moving solid surface is important in many extrusion processes, such as the aerodynamic extrusion of plastic sheets, cooling of a metallic plate in a cooling bath, the boundary layer along material handling conveyers, boundary layer along a liquid film in condensation processes, etc. Boundary layer flow and heat transfer over a fixed or moving flat plate in a viscous and incompressible fluid is well known. In view of these applications, Sakiadis [1] initiated the theoretical study of boundary layer on a continuous semi-infinite surface moving steadily through an otherwise quiescent fluid environment. An experimental and theoretical treatment was made for the flow past a continuous flat surface by Tsou et al. [2] who determined the heat transfer rates for certain values of the Prandtl number. Much theoretical work has been done on this problem since the pioneering papers by Sakiadis [1] and Flow of a micropolar fluid. Tsou et al. [2], and extensive references can be found in the papers by Magyari and Keller [3, 4], Liao and Pop [5], and Nazar et al. [6].

All the above investigations were restricted to Newtonian fluids. Due to the increasing importance in the processing industries (and elsewhere) of materials whose flow behavior in shear cannot be characterized by Newtonian relationships, a new stage in the evolution of fluid dynamics theory is in progress(Hassanien et al. [7]). Hoyt and Fabula [8], and Vogel and Patterson [9] conducted experiments with fluids containing small amounts of polymeric additives. These experiments indicated that fluids with additives display a reduction in skin friction near the surface of a rigid body. The Newtonian fluid mechanics cannot explain this phenomenon. Its extensions to non-Newtonian fluids are important for the thermal design of industrial equipments dealing with molten plastics, polymeric liquids, foodstuffs or slurries (Char and Chang[10]). Hence, considerable efforts have been directed toward this coupled, nonlinear boundary layer problem. The theory of micropolar fluids proposed by Eringen $[11,12]$ is capable of explaining the behavior of exotic lubricants, polymeric fluids, liquid crystals, animal bloods, colloidal and suspension solutions, etc., for which the classical Navier-Stokes theory is inadequate. A local microrotation vector together with the velocity vector describe the flow motion of such fluids. A comprehensive review of the subject and applications of micropolar fluid mechanics was given by Ariman et al. [13], Łukaszewicz [14] and Eringen [15].

Haque et al.[16] studied micropolar fluid behavior on steady magneto hydrodynamics free convection flow and mass transfer through a porous medium with heat and mass fluxes. Soret and dufour effects on mixed convection in a non- Darcy porous medium saturated with micropolar fluid was studied by Srinivascharya[17]. Olajuwon et,al.[18] 
investigated the effects of thermo-diffusion and thermal radiation on unsteady heat and mass transfer of free convective MHD micropolar fluid flow bounded by a semiinfinite porous plate in a rotating frame under the action of transverse magnetic field with suction. Mostafa et, al.[19] studied the effects of radiation on the boundary layer flow and heat transfer of an electrically conducting micropolar fluid over a continuously moving stretching surface embedded in a non-Darcian porous medium with a uniform magnetic field.

To the best of our knowledge, the use of Adomian Decomposition method to obtain the solution of hydromagnetic boundary layer micropolar fluid flow over a stretching surface embedded in a non Darcian medium with variable permeability has remained unexplored. The objective of the current work is to study the effect of various parameters that may occur on the velocity profile, microrotation and temperature profile.

\section{Materials and Methods}

\subsection{Model Formulation}

Consider a steady, two dimensional laminar flow of an incompressible, electrically conducting micropolar fluid over a continuously moving stretching surface embedded in a non-Dacian medium with variable permeability. The $x$-axis is taking along the stretching surface in the direction of the motion and $y$-axis is perpendicular to it. We assume that the velocity is proportional to its distance from the tin slit. A uniform magnetic field $B_{0}$ is imposed along $y$-axis. Then under the usual boundary layer approximations, the flow and heat transfer of a micro polar fluid in a non-Dacian medium with variable permeability effects included are governed by the following equations: Continuity equation:

$$
\frac{\partial u}{\partial x}+\frac{\partial v}{\partial y}=0
$$

Momentum Equation:

$$
u \frac{\partial u}{\partial x}+v \frac{\partial u}{\partial y}=v \frac{\partial^{2} u}{\partial y^{2}}+k_{1} \frac{\partial N}{\partial y}-\frac{\sigma B_{0}^{2}(x)}{\rho} u-\frac{v \varphi}{k} u
$$

Angular momentum:

$$
G_{1} \frac{\partial^{2} N}{\partial y^{2}}-2 N-\frac{\partial u}{\partial y}=0
$$

Energy equation:

$$
u \frac{\partial T}{\partial x}+v \frac{\partial T}{\partial y}=\frac{K}{\rho c_{p}} \frac{\partial^{2} T}{\partial y^{2}}+\frac{v}{c_{p}}\left(\frac{\partial u}{\partial y}\right)^{2}
$$

Subject to the boundary conditions:

$$
\begin{gathered}
y=0: u=a x, v=0, T=T_{w}, N=0, \\
y \rightarrow \infty: u \rightarrow 0, T \rightarrow T_{m} N \rightarrow 0,
\end{gathered}
$$

where $U$ is the apparent kinematic viscousity, $N$ is the microrotation component, $k_{1}=S / \rho$ is the coupling constant, $S$ is a constant characteristic of the fluid, $G_{1}$ is the microrotation constant, $\rho$ is the fluid density, $u$ and $v$ are the components of velocity along $x$ and $y$ directions, respectively, $\varphi$ is the porousity, $\mathrm{k}$ is the permeability of the medium, $T$ is the temperature of the fluid in the boundary layer, $T_{\infty}$ is the temperature of the fluid far away from the from the plate, $T_{w}$ is the temperature of the plate, $c_{p}$ is the specific heat capacity at constant pressure, $\sigma$ is the electrical conductivity, $B_{0}$ is an external magnetic field, $K$ is the thermal conductivity.

In other to transform our equations into cylindrical coordinate, we introduce the following transforms:

$$
\begin{gathered}
x \rightarrow r \cos \Theta, y \rightarrow r \sin \Theta, u \rightarrow u_{r}, v \rightarrow u_{\Theta} \\
\frac{\partial}{\partial y}=\frac{1}{r} \frac{\partial}{\partial \Theta}, \frac{\partial}{\partial x}=\frac{\partial}{\partial r}
\end{gathered}
$$

Substituting (6) into (1) to (5) we have the following: Continuity equation:

$$
\frac{1}{r}\left(\frac{\partial\left(r u_{r}\right)}{\partial r}+\frac{\partial u_{\Theta}}{\partial \Theta}\right)=0
$$

Momentum equation:

$$
\begin{gathered}
u_{r} \frac{\partial u_{r}}{\partial r}+\frac{u_{\Theta}}{r} \frac{\partial u_{r}}{\partial \Theta}= \\
\frac{v}{r} \frac{\partial}{\partial \Theta}\left(\frac{1}{r} \frac{\partial u_{r}}{\partial \Theta}\right)+\frac{k_{1}}{r} \frac{\partial N}{\partial \Theta}- \\
\frac{\sigma B_{0}^{2}(r)}{\rho} u_{r}-\frac{v \varphi}{k} u_{r}
\end{gathered}
$$

Angular momentum equation:

$$
\frac{G_{1}}{r} \frac{\partial}{\partial \Theta}\left(\frac{1}{r} \frac{\partial N}{\partial \Theta}\right)-2 N-\frac{1}{r} \frac{\partial u_{r}}{\partial \Theta}=0
$$

Energy equation:

$$
\begin{gathered}
u_{r} \frac{\partial T}{\partial r}+\frac{u_{\Theta}}{r} \frac{\partial T}{\partial \Theta}=\frac{\alpha}{r^{2}} \frac{\partial^{2} T}{\partial \Theta^{2}}+\frac{v}{c_{p}}\left(\frac{1}{r} \frac{\partial u_{r}}{\partial \Theta}\right)^{2} \\
\Theta=0: u_{r}=a r, u_{\Theta}=0, T=T_{w}, \\
N=0, \Theta \rightarrow \infty: u_{r} \rightarrow 0, T \rightarrow T_{\infty}, \\
N \rightarrow 0,
\end{gathered}
$$


Defining the dimensional stream function in the usual way such that $u_{r}=\frac{1}{r} \frac{\partial \psi}{\partial \Theta}$ and $u_{\Theta}=-\frac{\partial \psi}{\partial r}$ and using the following dimensionless variables Mostafa et al. [19]:

$$
\begin{gathered}
\eta=\left(\frac{a}{v}\right)^{\frac{1}{2}} r \Theta, \quad \psi=(a v)^{\frac{1}{2}} r f(\eta), \\
N=\left(\frac{a^{3}}{v}\right)^{\frac{1}{2}} \operatorname{rh}(\eta), \quad \theta(\eta)=\frac{T-T_{\infty}}{T_{w}-T_{\infty}}
\end{gathered}
$$

Substituting the expressions in (12) into (7) to (10), we have

$$
\begin{gathered}
f^{\prime \prime \prime}+f f^{\prime \prime}-\left(f^{\prime}\right)^{2}+L h^{\prime}(\eta)-\left(R+D^{-1}\right) f^{\prime}=0 \\
G h^{\prime \prime}-2 h-f^{\prime \prime}=0 \\
\frac{1}{p_{r}} \theta^{\prime \prime}+E C\left(f^{\prime \prime}\right)^{2}+f \theta^{\prime}-\theta=0
\end{gathered}
$$

Where $L=\frac{k_{1}}{v}, R=\frac{\sigma B_{0}{ }^{2}(r)}{a \rho}, D^{-1}=\frac{v \varphi}{a k} \quad G=\frac{G_{1} a}{v}$, $E c=\frac{a^{2} r^{2}}{c_{p}\left(T_{w}-T_{\infty}\right)}, p_{r}=\frac{\rho c_{p} v}{k}$, are coupling constant, magnetic parameter, inverse Darcy number, microrotation parameter, Eckert number, and prandtl number.

The corresponding boundary conditions are:

$$
\begin{aligned}
& f(0)=0, \quad f^{\prime}(0)=1, \theta(0)=1, h(0)=0, \\
& f^{\prime}(\infty)=0, \quad \theta(\infty)=0, \quad h(\infty)=0 .
\end{aligned}
$$

\subsubsection{Adomian Decomposition Method}

For the purpose of illustrating the method of Adomian decomposition we begin with the (deterministic) form $F(u)=g(\mathrm{t})$ where $F$ is a nonlinear ordinary differential operator with linear and nonlinear items. We could represent the linear term $L u$ where $L$ is the linear operator. In this case $L$ must be invertible which may have difficult Green's function a consequent difficult integration. Instead, we write the linear term $L u+R u$ where we choose $L$ as the highest-ordered derivative. Now $L^{-1}$ is simply $n$-fold integration for an $n^{\text {th }}$ order. The remainder of the linear operator is $R$ (in case where stochastic terms are present in linear operator, we can include a stochastic operator term $R u$ ). The nonlinear term is represented by $N u$.Thus, $L u+R u+N u=g$ we write $L^{-1} L u=L^{-1} \mathrm{~g}-L^{-1} R u$ $-L^{-1} N u$ For initial value problems we conveniently define $L^{-1}=\frac{d^{n}}{d t^{n}}$ as the $\mathrm{n}$ - fold definite integration operator from 0 to t. For the operator $L=\frac{d^{2}}{d t^{2}}$, for example we have,

$$
\begin{gathered}
L^{-1} L u=u-u(0)-t u^{\prime}(0) \therefore U=u(0)+L^{-1} \\
\mathrm{~g}-L^{-1} R u-L^{-1} N u
\end{gathered}
$$

For the same operator equation but now considering a boundary value problem, we let $L^{-1}$ be an indefinite integral and write $u=A+B t$ for the first two terms and evaluate $A$, $B$ from the given condition the first three terms are identified as $u_{0}$ in the assumed decomposition

$$
\mathrm{U}=\sum_{n=0}^{\infty} \boldsymbol{u}_{n}
$$

Finally, assuming $N u$ is analytic, we write $N u=$ $\sum_{n=0}^{\infty} \boldsymbol{A}_{\boldsymbol{n}}\left(\boldsymbol{u}_{\mathbf{0}} \boldsymbol{u}_{\boldsymbol{n}}\right)$ where the $\boldsymbol{A}_{\boldsymbol{n}}$ are specially generated Adomian polynomials for the specific nonlinearity.

\subsection{Method of Solution}

The nonlinear coupled differential equation (13) to (15) with boundary conditions (16) are solved using the ADM methods. If ADM applies on (13) to (15)

We define $L_{1}=\frac{d^{3}}{d \eta^{3}}$, and $L_{2}=\frac{d^{2}}{d \eta^{2}}$,

then

$$
\begin{gathered}
L_{1}[f]=-f f^{\prime \prime}+\left(f^{\prime}\right)^{2}-L h^{\prime}+\left(R+D^{-1}\right) f^{\prime} \\
L_{2}[h]=\frac{1}{G}\left(2 h+f^{\prime \prime}\right) \\
L_{2}[\theta]=-p_{r} E C\left(f^{\prime \prime}\right)^{2}-p_{r} f \theta^{\prime}+p_{r} \theta
\end{gathered}
$$

Applying inverse operator on (17) to $L_{1}^{-1} L_{1}[f]=-L_{1}^{-1}\left[f f^{\prime \prime}\right]+L_{1}^{-1}\left[\left(f^{\prime}\right)^{2}\right]$

$$
-L^{-1}{ }_{1}\left[L h^{\prime}\right]+L_{1}^{-1}\left[\left(R+D^{-1}\right) f^{\prime}\right]
$$

$$
L_{2}^{-1} L_{2}[h]=\frac{1}{G} L_{2}^{-1}\left[\left(2 h+f^{\prime \prime}\right)\right]
$$

$$
\begin{gathered}
L_{2}{ }^{-1} L_{2}[\theta]=-L_{2}{ }^{-1}\left[p_{r} E C\left(f^{\prime \prime}\right)^{2}\right]- \\
L_{2}{ }^{-1}\left[p_{r} f \theta^{\prime}\right]+L_{2}{ }^{-1}\left[p_{r} \theta\right]
\end{gathered}
$$

$$
\begin{gathered}
f=-L_{1}^{-1}\left[f f^{\prime \prime}\right]+L_{1}^{-1}\left[\left(f^{\prime}\right)^{2}\right]-L_{1}^{-1}\left[L h^{\prime}\right]+ \\
L_{1}^{-1}\left[\left(R+D^{-1}\right) f^{\prime}\right]+f(0)+\eta f^{\prime}(0)+\frac{\eta^{2}}{2} f^{\prime \prime}(0)
\end{gathered}
$$

$$
h=\frac{1}{G} L_{2}^{-1}\left(2 h+f^{\prime \prime}\right)+h(0)+\eta h^{\prime}(0)
$$




$$
\begin{aligned}
& \theta=-L_{2}^{-1}\left[p_{r} E C\left(f^{\prime \prime}\right)^{2}\right]-L_{2}^{-1}\left[p_{r} f \theta^{\prime}\right] \\
+ & L_{2}^{-1}\left[p_{r} \theta\right]+\theta(0)+\eta \theta^{\prime}(0)
\end{aligned}
$$

From the boundary conditions and taking $f^{\prime \prime}(0)=\alpha, h^{\prime}(0)=\beta$, and $\theta^{\prime}(0)=\gamma$, where $L_{1}^{-1}=\iiint(). d \eta d \eta d \eta$ and $L_{2}{ }^{-1}=\iint(). d \eta d \eta$

The ADM solution is obtain by:

$$
\begin{gathered}
\sum_{m=0}^{\infty} f_{m}(\eta)=\eta+\frac{\eta^{2}}{2} \alpha-L_{1}^{-1}\left[\sum_{m=0}^{\infty} A_{m}\right]+ \\
\sum_{n=0}^{\infty} h_{n}(\eta)=\eta \beta+\frac{1}{G} L_{2}^{-1}\left[\sum_{n=0}^{\infty}\left(2 h_{n}+f_{n}{ }^{\prime}\right)\right] \\
+L_{1}^{-1}\left[\sum_{m=0}^{\infty} B_{m}\right]-L_{1}^{-1}\left[\sum_{m=0}^{\infty} L h_{m}{ }^{\prime}\right]+L_{1}{ }^{-1}\left[\sum_{m=0}^{\infty}\left(R+D^{-1}\right) f^{\prime}{ }_{m}\right. \\
\sum_{n=0}^{\infty} \theta_{n}(\eta)=1+\eta \gamma-L_{2}^{-1}\left[p_{r} E C \sum_{n=0}^{\infty} C_{n}\right] \\
-L_{2}^{-1}\left[p_{r} \sum_{n=0}^{\infty} F_{n}\right]+L_{2}^{-1}\left[p_{r} \sum_{n=0}^{\infty} \theta_{n}\right]
\end{gathered}
$$

Where

$$
\begin{gathered}
A_{m}=\sum_{v=0}^{m} f_{m-v} f^{\prime \prime}{ }_{v} \\
B_{m}=\sum_{v=0}^{m} f^{\prime}{ }_{m-v} f^{\prime}{ }_{v} \\
C_{n}=\sum_{v=0}^{n} f^{\prime \prime}{ }_{n-v} f^{\prime \prime}{ }_{v} \\
F_{n}=\sum_{v=0}^{n} f_{n-v} \theta^{\prime}{ }_{v}
\end{gathered}
$$

from (26) to (28) we have:

$$
\begin{array}{r}
f_{0}(\eta)=\eta+\frac{\eta^{2} \alpha}{2} \\
h_{0}(\eta)=\beta \eta \\
\theta_{0}(\eta)=1+\gamma \eta
\end{array}
$$

For determination of other components of $f(\eta), h(\eta)$ and $\theta(\eta)$, we have:

$$
\begin{aligned}
& \sum_{m=0}^{\infty} f_{m+1}(\eta) \\
& =-L_{1}^{-1}\left[\sum_{m=0}^{\infty} A_{m}\right]+L_{1}^{-1}\left[\sum_{m=0}^{\infty} B_{m}\right]-L_{1}^{-1}\left[\sum_{m=0}^{\infty} L h_{m}{ }^{\prime}\right]+
\end{aligned}
$$

$$
\begin{gathered}
L_{1}^{-1}\left[\sum_{m=0}^{\infty}\left(R+D^{-1}\right) f^{\prime}{ }_{m}\right](36) \\
\sum_{n=0}^{\infty} h_{n+1}(\eta)=\frac{1}{G} L_{2}{ }^{-1}\left[\sum_{n=0}^{\infty}\left(2 h_{n}+f_{n}{ }^{\prime \prime}\right)\right] \\
\sum_{n=0}^{\infty} \theta_{n+1}(\eta)=-L_{2}{ }^{-1}\left[p_{r} E C \sum_{n=0}^{\infty} C_{n}\right]- \\
L_{2}{ }^{-1}\left[p_{r} \sum_{n=0}^{\infty} F_{n}\right]+L_{2}{ }^{-1}\left[p_{r} \sum_{n=0}^{\infty} \theta_{n}\right]
\end{gathered}
$$

The general solutions are:

$$
\begin{gathered}
f(\eta)=\sum_{m=0}^{\infty} f_{m}(\eta)=f_{0}+f_{1}+f_{2}+\ldots \\
h(\eta)=\sum_{n=0}^{\infty} h_{n}(\eta)=h_{0}+h_{1}+h_{2}+\ldots \\
\theta(\eta)=\sum_{n=0}^{\infty} \theta_{n}(\eta)=\theta_{0}+\theta_{1}+\theta_{2}+\ldots
\end{gathered}
$$

for conveniences, we used Maple-16 to compute the solutions and by Khedr et al. [20]

$$
\alpha=-0.616542, \beta=0.355330
$$

and

$$
\gamma=-0.249999
$$

\subsection{Results and Dicussion}

The system of non-linear coupled ordinary differential equations (13) to (15) with boundary conditions (16) has been solved using the Adomian Decomposition method and the results are in good agreement with that obtained by Mustafa et al. [19].

The effects of the Physical parameters R, D-1, Pr and Ec on the velocity profiles, micro rotations and temperature distributions are shown in Figures 1-8 above.

Figures 1 to 3 shows the velocity, angular velocity, and temperature distribution for various values of the magnetic parameter $R$, respectively, where $L=0.2, G=2$, $P_{r}=0.05$, and $E c=1.5$. As $R$ increases, the velocity profile and the micro rotation reduce, and the temperature profile increases. Application of a transverse magnetic field normal to the flow direction gives rise to a resistive drag-like force acting in a direction opposite to that flow. This has a tendency to reduce both the fluid velocity and angular velocity and increases the fluid temperature which is indicative from the decrease in the fluid velocity, angular velocity, and increases in the temperature profile.

Figures 4 to 6 displays the influence of the inverse Darcy number $D^{-1}$, where $R=1, G=2, L=0.2$, $P_{r}=0.05$, and $E c=1.5$ on the velocity profile, 
microrotation and the temperature profiles. As the Darcy number increases, both the velocity and the angular velocity reduce while the temperature profile increases.

Figures 7 to 8 depicts the influence of the $P_{r}$ and $E c$ on the temperature distributions while $R=1, G=2$, $L=0.2, D^{-1}=1$, and $E c=0.0$, and $E c=0.2$ respectively. It is observed that as $P_{r}$ increases for $E c=0$, temperature reduces and also for $E c=0.2$.

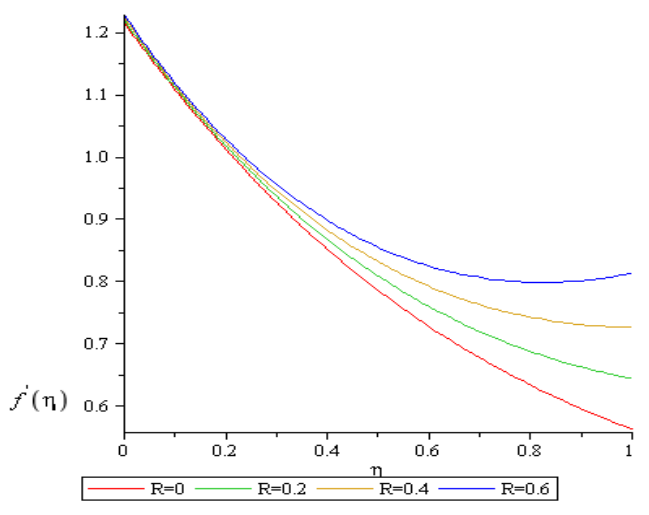

Figure 1. Graph of velocity profile $\left(f^{\prime}(\eta)\right)$ against $\eta$

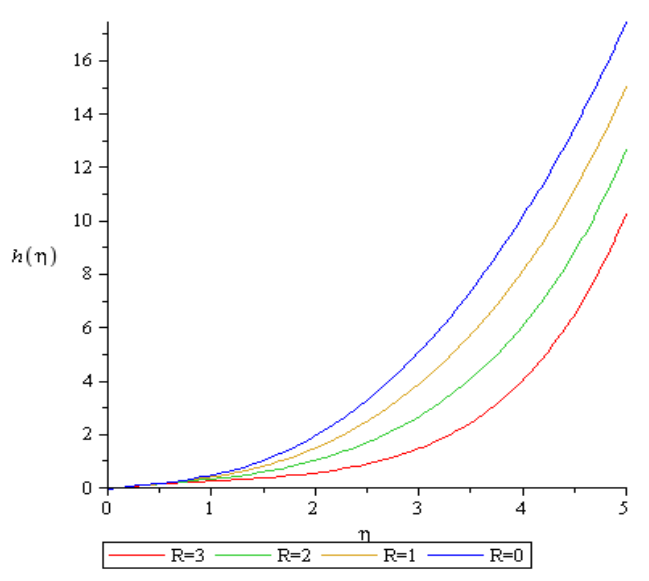

Figure 2. Graph of micro rotation $(h(\eta))$ against $\eta$

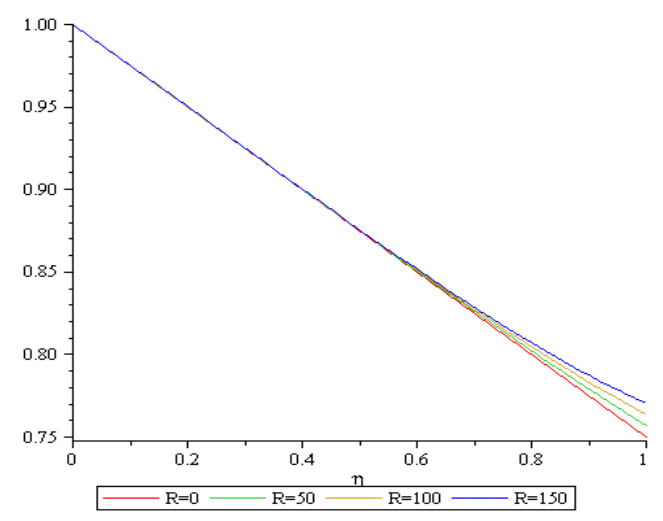

Figure 3. Graph of temperature profile $(\theta(\eta))$ against $\eta$

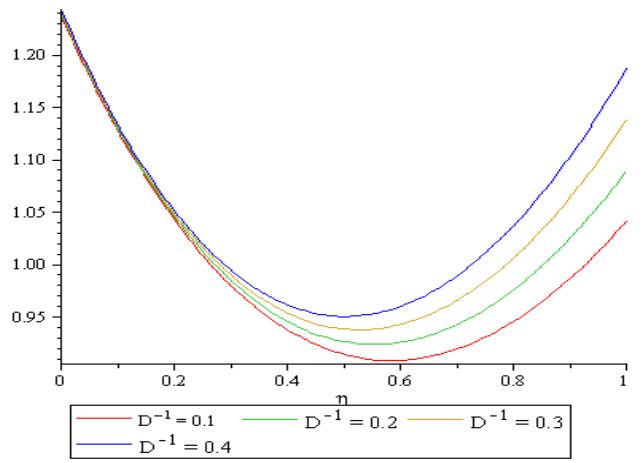

Figure 4. Graph of velocity profile $\left(f^{\prime}(\eta)\right)$ against $\eta$

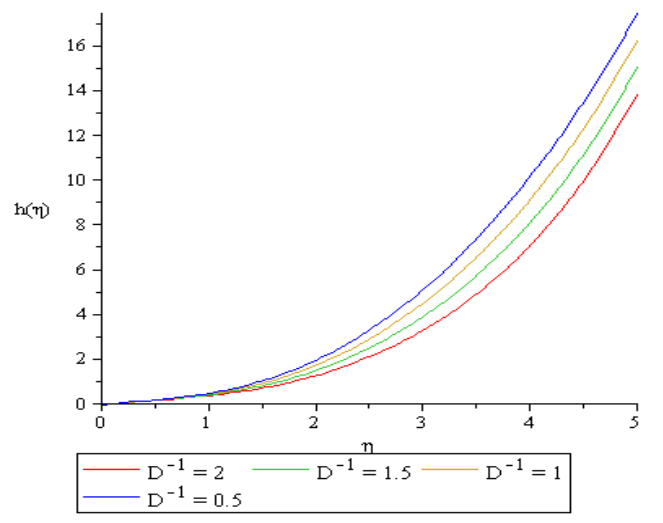

Figure 5. Graph of micro rotation $(h(\eta))$ against $\eta$

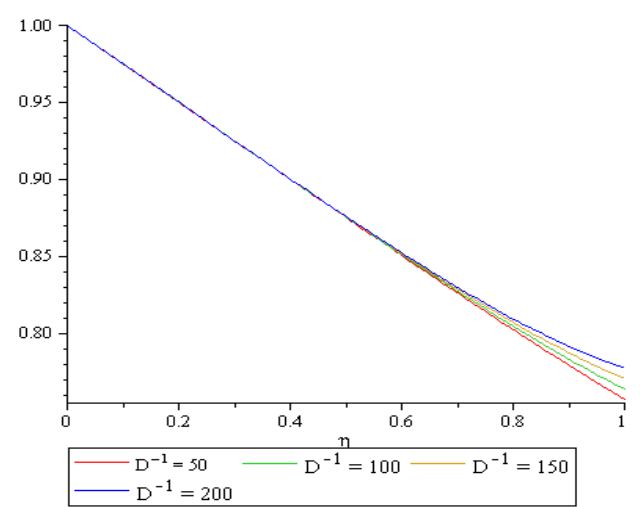

Figure 6. Graph of temperature profile $(\theta(\eta))$ against $\eta$

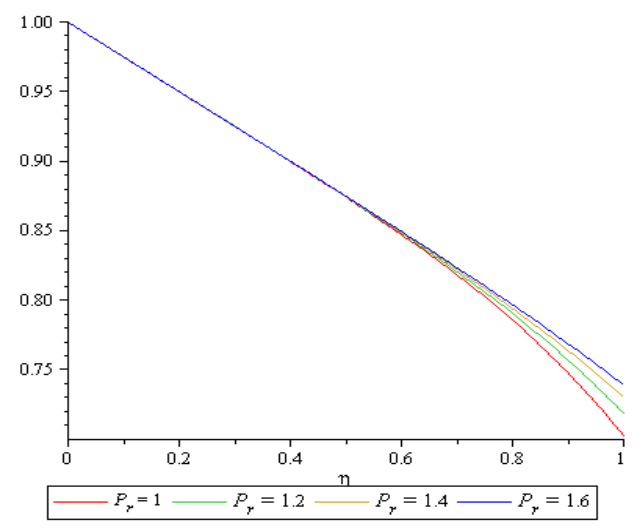

Figure 7. Graph of temperature profile $(\theta(\eta))$ against $\eta$ 


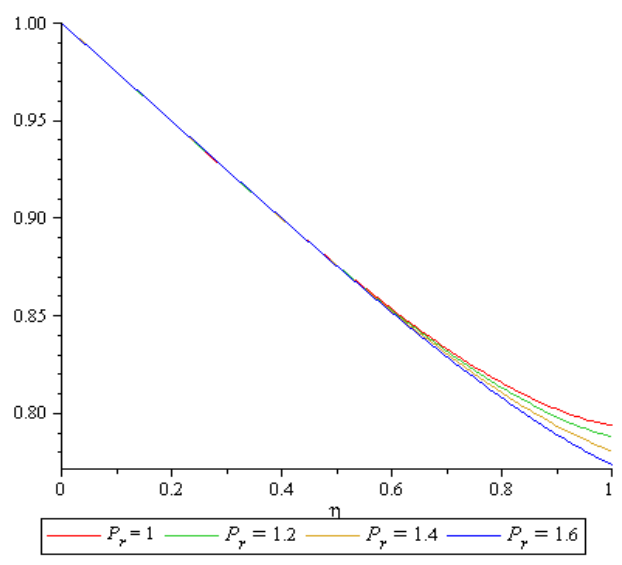

Figure 8. Graph of temperature profile $(\theta(\eta))$ against $\eta$

\section{Conclusion}

The problem of hydromagnetic boundary layer micropolar fluid flow over a stretching surface embeded in a non Darcian medium with variable permeability was considered. The governing equations for this problem were transformed into cylindrical coordinate system. The equation was also transformed using the appropriate similarity transformations. The resulting similarity equations were solved using the Adomian Decomposition method (ADM). Analytical evaluations were performed and the graphical results were obtained. It was observed that increase in the parameters $\mathrm{R}$ and $\mathrm{D}^{-1}$ reduces the velocity profiles and the microrotations, but enhances the temperature distributions. Increase in $P_{r}$ and $\mathrm{E}_{\mathrm{c}}$ also enhances the temperature profile.

\section{Acknowledgements}

We are very grateful to all the staff of Mathematics and Statistics Department, Federal University of Technology, Minna, Niger State, Nigeria.

\section{REFERENCES}

[1] B. C. Sakiadis, Boundary layers on continuous solid surfaces, AIChE. J., 7, 26-28, see also pp. 221-225 and 467-472, 1961.

[2] F. Tsou, E. Sparrow and R. Goldstein, Flow and heat transfer in the boundary layer on a continuous moving surface, Int. J. Heat Mass Transfer, 10, 219-235, 1967.

[3] E. Magyari and B. Keller, Heat and mass transfer in the boundary layers on an exponentially stretching continuous surface, J. Phys. D: Appl. Phys., 32, 577-585, 1999.

[4] E. Magyari and B. Keller, Exact solutions for self-similar boundary-layer flows induced by permeable stretching walls, Eur. J. Mech. B/Fluids, 19, 109-122, 2000.
[5] S. J. Liao and I. Pop, Explicit analytic solution for similarity boundary layer equations, Int. J. Heat Mass Transfer, 47, 75-85, 2004.

[6] R. Nazar, N. Amin and I. Pop, Unsteady boundary layer flow due to a stretching surface in a rotating fluid, Mech. Res. Comm., 31, 121-128, 2004.

[7] I. A. Hassanien, A. Shamardan, N. M. Moursy and R. S. R. Gorla, Flow and heat transfer in the boundary layer of a micropolar fluid on a continuous moving surface, Int. J. Numer. Methods Heat Fluid Flow, 9, 643-659, 1999.

[8] J. W. Hoyt and A. G. Fabula, The effect of additives on fluid friction, US Naval Ordinance Test Station Report, 1964.

[9] W. M. Vogel and A. M. Patterson, An experimental investigation of additives injected into the boundary layer of an underwater body, Pacific Naval Lab. of the Defense Res. Board of Canada, Report 64-2, 1964.

[10] M. I. Char and C. L. Chang, Effect of wall conduction on natural convection flow of micropolar fluids along a flat plate, Int. J. Heat and Mass Transfer, 40, 3641-3652, 1997.

[11] A. C. Eringen, Theory of micropolar fluids, J. Math. Mech., 16, 1-18, 1966.

[12] A. C. Eringen, Theory of thermomicrofluids, J. Math. Analy. Appl., 38, 480-496, 1972.

[13] T. Ariman, M. A. Turk and N. D. Sylvester, Microcontinuum fluid mechanics - a review, Int. J. Engng. Sci., 11, 905-930, 1973.

[14] G. Łukaszewicz, Micropolar Fluids: Theory and Application, Birkhäuser, Basel 1999.

[15] A. C. Eringen, Microcontinum Field Theories. II: Fluent Media, Springer, New York 2001.

[16] Haque Md Z, Alam Md M, Ferdows M, Postelnicu A. Micropolar fluid behaviors on steady MHD free convection flow and mass transfer with constant heat and mass fluxes, joule heating and viscous dissipation. J.King Saud Univ.Engg.Sci.doi:10.1016/j.jksues.2011.02.003, 2011.

[17] Srinivasacharya.D., Ramreddy. C.,Soret and Dufour effect on mixed convection in a non-Darcy porous medium saturated with micropolar fluid, Non-analysis modelling and control, vol.16,No.1, 100-115,2011.

[18] B.I. Olajuwon and J.I. Oahimire, Unsteady Free Convection Heat And Mass Transfer In An Mhd Micropolar Fluid InThe Presence Of Thermo Diffusion And Thermal Radiation. International Journal of Pure and Applied Mathematics, Volume 84: No 2. 15-37, 2013.

[19] A. A. M. Mostafa, A. M. Mahamoud, S. E. Waheed, Hydromagnetic boundary later micropolar fluid flow over a stretching surface embedded in a non-Darcian medium with radiation, J. Mathematical problems in Engineering, volume 2006: 1-10, 2006.

[20] M.-E.M. Khedr, A. J. Chamkha, M. Bayomi, MHD Flow of a Micropolar Fluid past a Stretched Permeable Surface with Heat Generation or Absorption, Nonlinear Analysis: Modelling and Control, Vol. 14: No. 1, 27-40, 2009 